\title{
Photonic crystal nanocavities with quantum well or quantum dot active material
}

\section{Tomoyuki Yoshie, Marko Loncar, Koichi Okamoto, Yueming Qiu, Oleg B. Shchekin, et al.}

Tomoyuki Yoshie, Marko Loncar, Koichi Okamoto, Yueming Qiu, Oleg B. Shchekin, Hao Chen, Dennis G. Deppe, Axel Scherer, "Photonic crystal nanocavities with quantum well or quantum dot active material," Proc. SPIE 5360, Photonic Crystal Materials and Devices II, (9 July 2004); doi: $10.1117 / 12.525869$

SPIE Event: Integrated Optoelectronic Devices 2004, 2004, San Jose, CA, United States 


\title{
Photonic crystal nanocavities with quantum well or quantum dot active material
}

\author{
Tomoyuki Yoshie $^{a}$, Marko Lončar ${ }^{a}$, Koichi Okamoto $^{a}$, Yueming Qiu $^{b}$, Oleg B. Shchekin ${ }^{c}$, Hao \\ Chen $^{c}$, Dennis G. Deppe ${ }^{c}$, and Axel Scherer ${ }^{a}$ \\ ${ }^{a}$ Department of Electrical Engineering, Caltech, Pasadena CA 91125-9300 U.S.A. \\ ${ }^{b}$ Jet Propulsion Laboratory, Caltech, Pasadena CA 91109 U.S.A. \\ ${ }^{c}$ Microelectronics Research Center, Department of Electrical and Computer Engineering, \\ the University of Texas at Austin, Austin TX, 78712-1084 U.S.A.
}

\begin{abstract}
We have investigated the miniaturization of photonic devices for ultimate photon localization, and have demonstrated two-dimensional photonic crystal nanolasers with two important quantum nanostructures- quantum wells (QWs) and quantum dots (QDs). Photonic crystal cavities with QW active material are simple, but powerful nanolasers to produce intense laser output for signal processing. On the other hand, when located in a highquality factor $(\mathrm{Q})$ nanocavity, because QD(s) strongly couple with the intense optical field, QD photonic crystal cavities are expected to be good experimental setups to study cavity quantum electrodynamics, in addition to high speed and compact laser sources. Our photonic crystal nanolasers have showed as small thresholds as $0.12 \mathrm{~mW}$ and $0.22 \mathrm{~mW}$ for QD-photonic crystal lasers and QW- photonic crystal lasers, respectively, by proper cavity designs and nanofabrication. For QD-photonic crystal lasers, whispering gallery modes in square lattice were used together with coupled cavity designs and, for QW-photonic crystal lasers, quadrapole modes in triangular lattice with fractional edge dislocations were used to produce high- $Q$ modes with small mode volume.
\end{abstract}

Keywords: photonic crystal, laser, quantum well, quantum dot

\section{INTRODUCTION}

Enhancement and inhibition of radiation process were predicted to occur by Purcell. ${ }^{1}$ The modification of the radiation process is understood by weak light-matter coupling in a frame of cavity quantum electrodynamics. Further optical confinement in small space causes strong interaction of light with matter in the cavity. Photonic crystal $(\mathrm{PC})^{2,3}$ cavities are believed to provide such ultimate photon localization. Such PC cavities can be compact building blocks, which will be used in future integrated nanophotonic systems. One of the most notable characters of $\mathrm{PC}$ cavities is that the mode profile and the quality $(Q)$ factors are geometrically controlled. When combined with active material such as quantum wells (QWs) and quantum dots (QDs), simple defect cavity designs provide compact lasers. The first photonic crystal lasers ${ }^{4}$ were demonstrated on InGaAsP multi-QW structures. QW-PC lasers are simple, but powerful light sources. On the other hand, lasing actions from QD-PC nanocavities are difficult due to the small gain generation, but they have some advantages as efficient light sources over QW-PC lasers. They are (1)small surface recombination rate, (2) high spontaneous emission coupling $(\beta)$ factor, (3) small absorption loss by active materials in the surroundings, and (4) high speed signal modulation with small chirping.

We describe simple designs of planar photonic crystal nanolasers with quantum well or quantum dot active materials, and demonstrate low-threshold laser actions from such quantum active nano-materials.

\section{QUANTUM WELL PHOTONIC CRYSTAL NANOLASERS}

Planar photonic crystal nanocavities are fabricated in InGaAsP quantum well material, which consists of four $9 \mathrm{~nm}$ thick, compressively strained, quantum wells $(\lambda=1550 \mathrm{~nm})$, separated by $20 \mathrm{~nm}$ InGaAsP barriers

Further author information: (Send correspondence to T.Y.)

T.Y.: E-mail: yoshie@caltech.edu, Telephone: 16263952207 


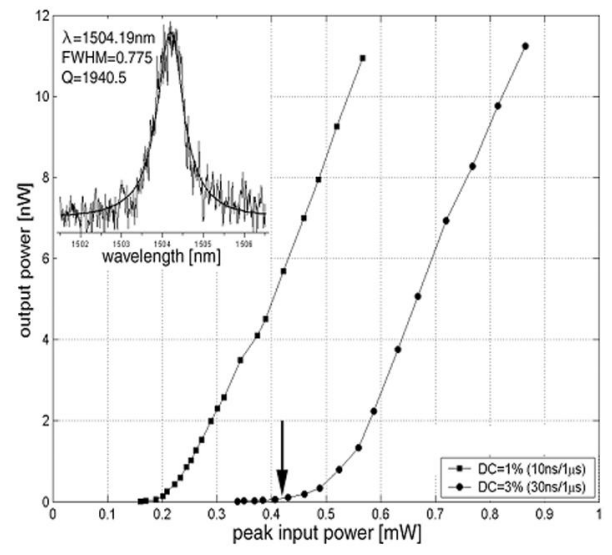

Figure 1. Collected peak output power of QW nanolasers as a function of input peak pump power. The duty cycle is $1 \%$ and $3 \%$ for black squares and black circles. The measurements were conducted at room temperature.

$(\lambda=1220 \mathrm{~nm})$. This active material is grown in the center of a $330 \mathrm{~nm}$ thick InGaAsP slab. An InGaAs layer is introduced between the sacrificial layer and the InP substrate to automatically stop wet-etching at the layer. An etching mask is $40 \mathrm{~nm} \mathrm{Au}$ on a top of $100 \mathrm{~nm} \mathrm{SiON}$, deposited using plasma-enhanced chemical vapor deposition. $150 \mathrm{~nm}$ poly methylmethacrylate (PMMA) electron beam resist was used to define patterns for electron-beam lithography. We use argon ion milling to transfer the patterns through the Au metal mask, and follow this step by a $\mathrm{C}_{2} \mathrm{~F}_{6}$ reactive ion etching (RIE) to transfer the mask from the Au into the $\mathrm{SiON}$. $\mathrm{Cl}_{2}$-based inductively coupled plasma RIE is finally used to transfer the patterns from the SiON layer into the InGaAsP slab. The mask is removed in a hydrofluoric acid and the InGaAsP membrane is released from the substrate by wet etching in $\mathrm{HCl}$ solution. The final structure is a free standing membrane supported at one side. Each cluster consists of six different cavities that have received the same electron dose during the electron beam lithography step and, therefore, should have similar hole size $(r)$ and lattice constant $(a)$. The only difference between cavities within a pattern is the value of the elongation parameter $p$ that assumed values in the range $p=0 \sim 0.25 a$. The periodicity of the structure characterized here was $a \sim 435 \mathrm{~nm}$ and a hole radius $r \sim 138 \mathrm{~nm}$. This combination of geometries leads to a relative thickness of $d / a=0.76$ and relative hole size of $r / a=0.32$. Using three-dimensional finite difference time domain code (3D-FDTD), we have found that in this case, the photonic band gap is located in the range $a / \lambda=0.253 \sim 0.345$. The structures were optically pumped using $10 \sim 30 \mathrm{nsec}$ long pulses in a period of $1 \mu \mathrm{sec}$ from a 830nm semiconductor laser diode. The pump beam was focused through a $100 \times$ objective lens onto the sample surface to obtain a $3 \mu \mathrm{m}$ spot size. The emission from the cavities is collected through the same lens, and the spectrum of the emitted light signal is detected with an optical spectrum analyzer.

The devices were tested for lasing. ${ }^{5}$ Poor thermal heat sinking was expected for our membrane resonators, since the cavities are located in free standing membranes. Thus, we have pumped the structures with limited duty cycles $(\mathrm{DC}<3 \%)$. Lasing was observed in our cavities for several $\mathrm{p} 4(p / a=0.20)$ and $\mathrm{p} 5(p / a=0.25)$ structures, with different $r / a$ parameters and defect hole sizes. In some cases, lasing could also be observed in $\mathrm{p} 3$ $\operatorname{cavities}(p / a=0.15)$. In Fig.1, we show the dependence of the output power on the input power for one of the lasing photonic crystal nanocavities. Threshold powers as low as $P_{t h}=214 \mu \mathrm{W}$ were observed when this laser was pumped with approximately $1 \%$ duty cycle.

Our 3D-FDTD modeling predicts that the lasing modes have small mode volumes. To investigate the field profiles of lasing modes, we employed a near-field scanning optical microscope (NSOM). ${ }^{6}$ An optical fiber tip is placed close to a sample surface by shear force feedback, and a He-Ne laser $(633 \mathrm{~nm})$ and a diode laser (780nm) were used as $\mathrm{cw}$ excitation laser sources. Used samples have fractional edge dislocations in a single defect triangular lattice. A sample was selected such that only two localized modes (high- $Q$ quadrapole mode and low- $Q$ dipole mode) are seen in an InGaAsP QW emission range by $\mu$-photoluminescence. The emission was detected by a high-gain detector through a objective lens placed on a top of the sample. Metal coating on sides of the optical fiber tip helps suppression of signal detection of propagation modes. Figure 2 (a) is a typical 

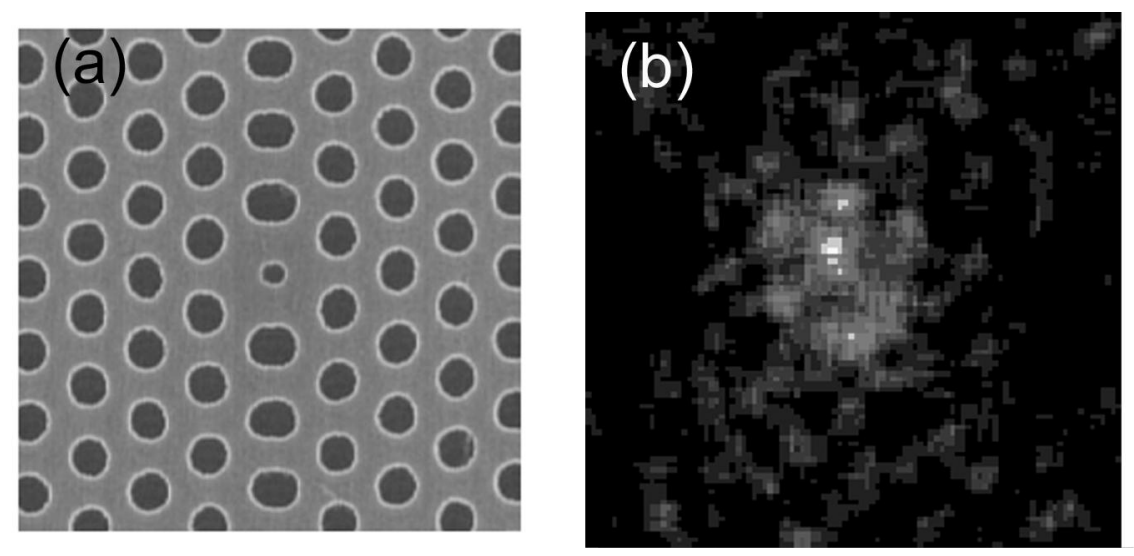

Figure 2. Panel (a) is a scanning electron microscopy image of a typical sample measured for NSOM. Panel (b) is a near-field scanning microscopy image of a superposition of orthogonal dipole modes. An NSOM tip was used to excite the modes.

sample image taken by a scanning electron microscope. Figure 2 (b) is an NSOM image of the sample. Without any filters, the observed image is considered to be a superposition of the two modes. We measured $\lambda / \Delta \lambda=2,000$ around the threshold and realized laser action with low threshold of $220 \mu \mathrm{W}$ in the design with $r^{\prime} / a=0.18\left(r^{\prime}\right.$ : defect air hole size). The bright spot was found in this image located at the center of the PC structures, matching the positions of the defect cavities. The small spots indicate that, indeed, the modes have small mode volumes as expected from our modeling. The NSOM images were very similar with a superposition of two field-profiles obtained by our 3D-FDTD modeling. An intensity decrease in the observed envelop function from the center defect to surroundings indicates evanescent decaying by photonic bandgap confinement of light. In the NSOM image, tighter optical confinements are observed along the horizontal direction than along the vertical direction. This results from better lateral confinements in high- $Q$ quadrapole modes than in low- $Q$ dipole modes.

\section{QUANTUM DOT PHOTONIC CRYSTAL NANOLASERS}

Quantum dot photonic crystal nanolasers were fabricated by using self-assembled InAs QDs and localized modes in planar square lattice. Five stacked self-assembled InAs QDs layers were grown by molecular beam epitaxy. The QD density in our samples is $5 \times 10^{10} / \mathrm{cm}^{2}$ in total and the QD layers were separated by 30nm GaAs layers. $\mathrm{Al}_{0.2} \mathrm{Ga}_{0.8} \mathrm{As}$ is used for cladding layers to form thin slab waveguides, which are deposited on an $800 \mathrm{~nm}$ $\mathrm{Al}_{0.94} \mathrm{Ga}_{0.06} \mathrm{As}$ sacrificial layer on a top of GaAs substrate. To define 200nm-thick 2D-PC slab cavities, electronbeam lithography, $\mathrm{Cl}_{2}$-based chemically assisted ion-beam etching, oxidation of $\mathrm{Al}_{0.94} \mathrm{Ga}_{0.06} \mathrm{As}$ layer, and wet etching of $\mathrm{Al}_{x} \mathrm{O}_{y}$ layer were performed in regular sequence. The detailed fabrication steps can be found elsewhere. $^{7}$ Many geometries of single-, two-, and four-defect coupled cavities were fabricated within photonic crystals with lattice sizes of $31 \times 31,31 \times 33$, and $33 \times 33$, respectively.

A pulsed 780nm semiconductor laser diodes was used to measure luminescence spectra and collected resonance power as a function of input pump power (L-L curves). Twenty nanosecond pulses with $2.86 \%$ duty cycle were applied to the pumping laser diodes. The pump light was confirmed to have good pulse shapes in time domain by a fast detector and a digital oscilloscope. The pumping beam diameter was varied up to $4 \mu \mathrm{m}$.

Figure 3-(a) shows two L-L curves taken from two- and four-defect coupled cavities. Each of these cavities exhibits distinct threshold and linear increase in output power above threshold. Threshold pump powers of $120 \mu \mathrm{W}$ and $370 \mu \mathrm{W}$ are measured for two- and four-defect coupled cavities, respectively. Considering filling factors and transmission of pump light at air/semiconductor interfaces, the pump light power in the membranes would be approximately only $15 \%$ of incident pump power with perfect absorption by cavities. Fifteen percentages of threshold incident pump powers are $18 \mu \mathrm{W}$ and $56 \mu \mathrm{W}$ for two-defect and four-defect coupled cavities, respectively. Actual absorbed pump power would be smaller than this estimation due to imperfect absorption. These facts 

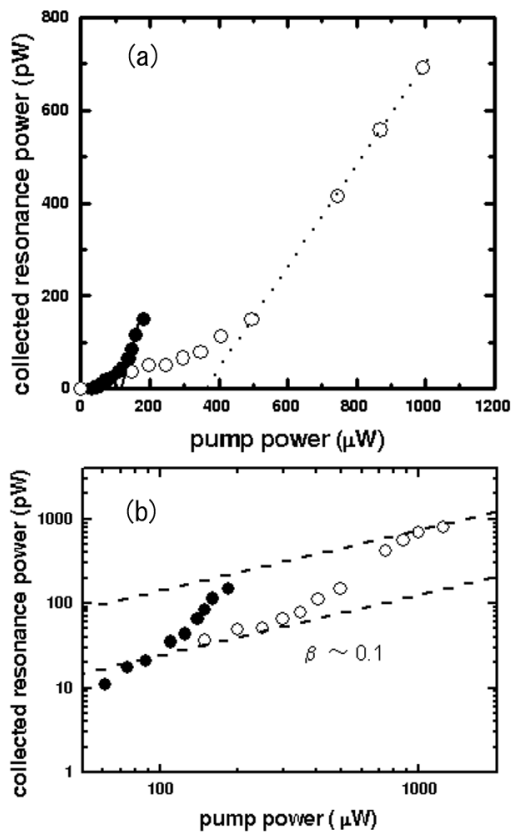

Figure 3. Collected peak output power of nanolasers as a function of input peak pump power for quantum dot photonic crystal nanolasers. Black and white circles are take from two-defect and four-defect coupled cavities, respectively. Panel (a)is a linear-linear graph, and (b) is a log-log graph to show approximate $\beta$ factors. The duty cycle is $3 \%$, and the pulse width is 20nanoseconds. The measurements were conducted at room temperature. The $r / a \mathrm{~s}$ are 0.34 and 0.38 , and $a$ s are $400 \mathrm{~nm}$ and $420 \mathrm{~nm}$ for two- and four-defect coupled cavities, respectively. $d$ is $200 \mathrm{~nm}$.

allow us to believe that our nanolasers are operated by very small power and that sub- $\mu \mathrm{W}$ threshold lasers could be constructed by improvement of fabrication. Figure 3(b) shows a log-log plot of output power as a function of input pump power. From the figure, it can be derived that the spontaneous emission coupling $(\beta)$ factors are as small as 0.1 . This indicates that $10 \%$ of emission from quantum dot contributing to the lasing mode couples with the lasing modes and $90 \%$ of the emission couples with the rest of optical modes.

Figure 4 shows luminescence spectra from a four-defects coupled cavity. Below threshold (Fig.4 (a)), the resonance cannot be clearly observed. Close to threshold, nonlinear amplification of emission is seen (Fig.4 (b)). Above threshold (Fig.4 (c)), the resonance intensity increases significantly. The lasing wavelength of $1328 \mathrm{~nm}$ matches the QDs ground state emission. The lasing frequency of $a / \lambda=0.316$ in this laser also matches the analyzed mode frequency of two-defect coupled cavities. Spectral line widths decreased from $1 \sim 2 \mathrm{~nm}$ (below threshold) to less than $0.2 \mathrm{~nm}$ (above threshold). With moderate CW pumping, the resonance showed $\lambda / \Delta \lambda=2100$. The combination of distinct threshold in the $L_{\text {pump }}-L_{\text {out }}$ curves and line width narrowing both indicate that the cavities are definitely lasing. ${ }^{8} \quad$ For two-defect coupled cavities, the resonance was measured at $a / \lambda=0.3$. The simulated mode volumes are $0.8 \times(\lambda / n)^{3}$ and $1 \times(\lambda / n)^{3}$, respectively, by using geometric parameters of lased PC samples. Single defect cavities did not lase in our experiments, probably due to a lack of sufficient gain from the QDs, but the single defect cavities have strong possibilities of lasing from our simulation results.

The number of QDs contributing to the lasing is calculated. The approximated formula is derived by starting from the threshold condition shown in a reference ${ }^{9}$ with an assumption that material permittivity $\epsilon(r)$ is almost uniform. The assumption seems to contradict with photonic crystals, but this is a good approximation to obtain an approximate point of threshold. In addition, the assumption is also good on our structures, in which all gain material is located on the photonic crystal slab, i.e., $\epsilon(r)=$ constant on all gain material. Then, we start from 


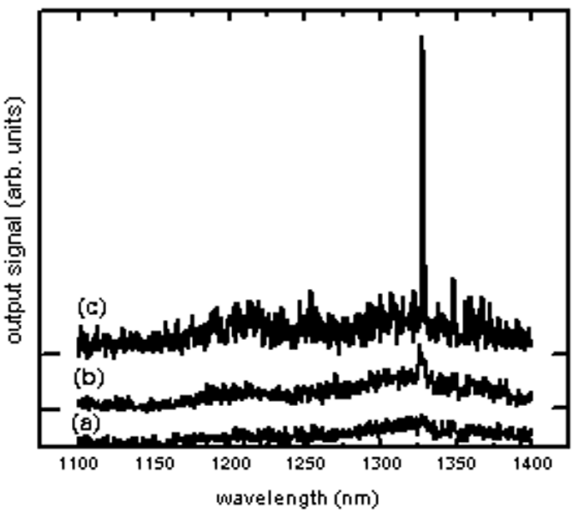

Figure 4. Spectra taken at three pump power levels for four-defect coupled cavity with quantum dots. The duty cycle is $3 \%$, and the pulse width is 20 nsec. The measurements were conducted at room temperature.(a) $140 \mu W$, (b) $250 \mu W$, (c) $990 \mu \mathrm{W}$

a threshold condition for a normal mode $E_{l}(\vec{r})$ as follows ${ }^{9}$ :

$$
\begin{gathered}
\left(\omega_{l}^{2}-\omega^{2}\right)+i \frac{\sigma \omega}{\epsilon}=\frac{\omega^{2} \epsilon_{0} f}{\epsilon}\left(\chi^{\prime}-i \chi^{\prime \prime}\right) \\
\int_{V} d r^{3} E_{l}(\vec{r}) \cdot E_{l}(\vec{r})=1 \\
f=\int_{V_{\text {active }}} d r^{3} E_{l}(\vec{r}) \cdot E_{l}(\vec{r})
\end{gathered}
$$

where $\omega=2 \pi \nu, \omega_{l}=2 \pi \nu_{l}, \sigma=\epsilon \omega / Q, \chi=\chi^{\prime}-i \chi^{\prime \prime}$ are lasing angular frequency, resonance angular frequency, conductivity and complex susceptibility. $V_{\text {active }}$ is a volume where gain or loss is generated in a mode of interest.

The equation(1) gives a threshold condition containing $Q$ factor and lasing wavelength at threshold and a lasing wavelength condition. Imaginary part of susceptibility is given by

$$
\chi^{\prime \prime}=\frac{\mu^{2}}{2 \epsilon_{0} \hbar} \Delta N \frac{\Delta \nu / 2 \pi}{\left(\nu-\nu_{i}\right)^{2}+\left(\frac{\Delta \nu}{2}\right)^{2}}
$$

where $\epsilon_{0}$ and $\Delta N$ are permittivity in vacuum and the population inversion number per unit volume. The eqn.(4) is Lorentzian-shaped to represent gain from a single quantum dot.

After plugging the eqn.(4) into an imaginary part of eqn. (1), the following threshold condition is derived for a single quantum dot as

$$
\frac{\omega_{l}}{Q}=\sum_{m=1}^{M} \frac{g \omega \mu_{m}^{2} \Delta \rho_{m}}{2 \varepsilon V_{o} C_{q}} \frac{\hbar \Delta \omega_{m}}{\left(\hbar \omega-\hbar \omega_{m}\right)^{2}+\left(\frac{\hbar \Delta \omega_{i, m}}{2}\right)^{2}}
$$

and for $N$ quantum dots as

$$
\frac{\omega_{l}}{Q}=\sum_{i=1}^{N} \sum_{m=1}^{M} \frac{g_{i} \omega \mu_{i, m}^{2} \Delta \rho_{i, m}}{2 \varepsilon V_{o} C_{q}} \frac{\hbar \Delta \omega_{i, m}}{\left(\hbar \omega-\hbar \omega_{i, m}\right)^{2}+\left(\frac{\hbar \Delta \omega_{i, m}}{2}\right)^{2}}
$$

where

$$
\frac{1}{C_{q}}=\frac{\left|E_{l}(\vec{r}) \cdot E_{l}(\vec{r})\right|}{\max \left[E_{l}(\vec{r}) \cdot E_{l}(\vec{r})\right]}
$$




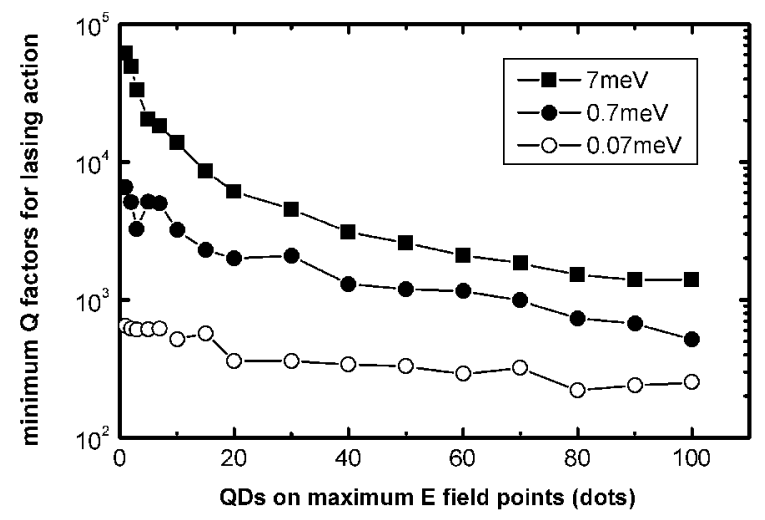

Figure 5. Minimum $Q$-factors to obtain lasing as a function of the number of QDs locating on maximum optical field points. Calculations are made for three homogeneous broadening: $7 \mathrm{meV}, 0.7 \mathrm{meV}$ and $0.07 \mathrm{meV}$. Due to random pick-up of QDs emission energy in Gaussian, the $Q$-factors needed for lasing are fluctuated. $n=3.4, C=1$, and homogeneous broadening is $40 \mathrm{meV}$.

$$
V_{o}=\frac{\int_{V} d r^{3} E_{l}(\vec{r}) \cdot E_{l}(\vec{r})}{\max \left[E_{l}(\vec{r}) \cdot E_{l}(\vec{r})\right]}
$$

, and $g$ and $\Delta \rho$ are degeneracy of states and inversion factor of the transition. $\omega \simeq \omega_{l}$ was also assumed because of the following condition explaining "the frequency pulling" of lasers.

$$
\omega=\frac{\omega_{l}}{\sqrt{1+\frac{\epsilon_{0} f}{\epsilon} \chi^{\prime}}} \simeq \omega_{l}\left(1-\frac{\epsilon_{0} f}{2 \epsilon} \chi^{\prime}\right)
$$

We define that effective number of QDs $\left(N_{\text {lasing }}\right)$ to obtain lasing action as the number of QDs located at maximum electric field, i.e., $C_{q}=1$. In order to obtain $N_{\text {lasing }}$, we assumed the conditions such as $\Delta \rho=1, \epsilon=$ $3.4^{2} \epsilon_{o}, V_{\text {cavity }}=C(\lambda / n)^{3}$, and squared QD dipole moment $\mu^{2}=1 \times 10^{-56}\left[\mathrm{C}^{2} \cdot \mathrm{m}^{2}\right] .{ }^{10}$ In addition, we randomly picked up the energy of each quantum dot in a Gaussian distribution, representing inhomogeneous broadening. An inhomogeneous broadening used in the following calculations is $40 \mathrm{meV}$.

Figure 5 shows the minimum $Q$-factors as a function of the number of QDs on the maximum $E$ field points and homogeneous broadening of a single QD to obtain lasing under the above assumptions. The values are numerically obtained. If $0<\Delta \rho<1$ or $0<C_{q}<1$, the minimum $Q$-factor can be increased by a factor of $1 /\left(\Delta \rho C_{q}\right)$. Assuming that our lasers have $Q$-factors of $2000, \Delta \rho=0.7$, homogeneous broadening of $7 \mathrm{meV}$ and $C=1.0$ for four-defect coupled cavities, the effective number of QDs are around 80. This is reasonable values, considering that the lasing mode has more QDs and the positions of QDs are random.

\section{SINGLE QUANTUM DOT LASERS}

Single QD lasers, which are analogous to a one atom maser (micromaser), ${ }^{11}$ are one of the ultimate laser sources. One atom masers emit a coherent electromagnetic wave in, typically, the microwave region, resulting from strong atom-cavity coupling. For example, three level system of single Rubidium 85 is located in a high- $Q$ cavity to create population inversion, and $63 P_{3 / 2}-61 P_{5 / 2}$ transition emits coherent $21.45 \mathrm{GHz}$ microwave.

When located in a high- $Q$ cavity, the single quantum dot functions as an optical gain source with sharp linewidth like the atom does. It is desirable to use a solid-state QD instead of an atom beam for photonic circuits and quantum information systems, requiring rich resources of the state of art telecommunication technology. Using the equations in the previous section, we can derive what high $Q$ factors are needed to obtain lasing action from a single QD in our PC cavities. Figure 5 also include minimum $Q$-factors to obtain single QD lasers. At room temperature homogeneous broadening is likely to be around $7 \mathrm{meV}$, and the minimum $Q$-factors for single 


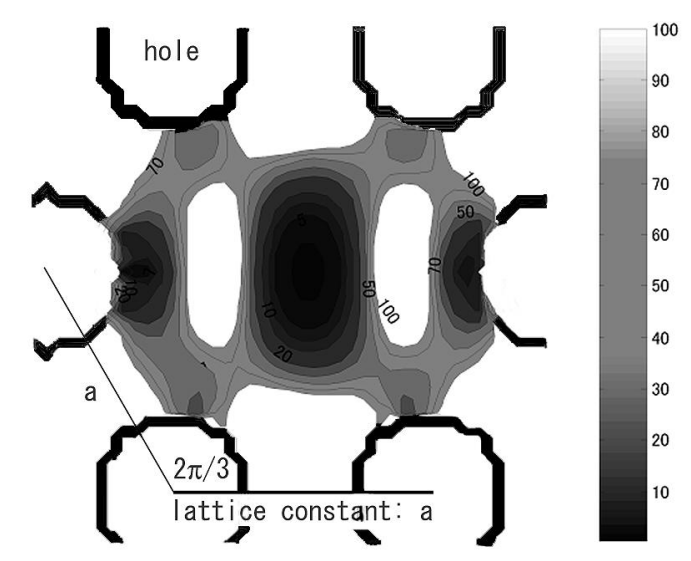

Figure 6. Mapping of $C_{q}$ value dependence on location of QDs for dipole mode in triangular lattice, which has small $Q$-factors. Six circles represent triangular lattice holes in dielectric slab. The figure center is a missing lattice point, which supports dipole modes. A distance between adjacent circles on horizontal axis is a lattice constant $(a)$ of triangular lattice.

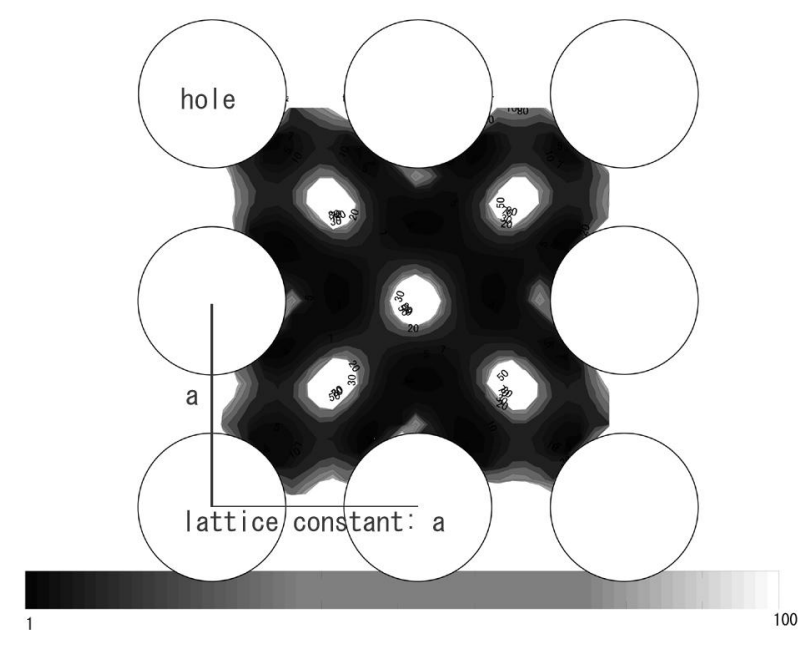

Figure 7. Mapping of $C_{q}$ value dependence on location of QDs for whispering gallery mode in square lattice, which has high $Q$-factors. Eight circles represent square lattice holes in dielectric slab, and the figure center is a missing lattice point, which supports whispering gallery modes. A distance between adjacent circles is a lattice constant $(a)$ of square lattice.

QD lasing are at least 60,000 , which is smaller than predicted maximum $Q$-factors of WGMs generated in single defect square lattice holes. By decreasing sample temperature to $77 \mathrm{~K}$ or cooler, the homogeneous broadening is easily narrowed to less than $1 \mathrm{meV}$. Then lasings are more likely to occur since, for homogeneous broadening of $0.7 \mathrm{meV}, 6,600$ is a minimum $Q$-factor for single $\mathrm{QD}$ lasing.

A decrease in QD density in samples in order to pursue single QD lasing makes it unlikely that the single QD is located at the maximum $E$ field points. In order to know the single QD position effects, $C_{q}$ value mapping over PC structures are obtained by using two PC modes of dipole modes in triangular lattice and WGMs in square lattice. The analyzed dipole modes have $Q_{v}=300$ and $V_{\text {mode }}=0.22(\lambda / n)^{3}$. Figure 6 shows $C_{q}$ mapping over a middle slice of PC slabs. It can be noticed that the center is the best point to put a single QD for this mode. Necessary $Q$-factors by eqn.(5) are $15,000 \times C_{q}$ and $1,600 \times C_{q}(\gg 300)$ for homogeneous broadening of $7 \mathrm{meV}$ and $0.7 \mathrm{meV}$, respectively. Therefore, even though we succeed to locate a single QD position on maximum $E$ field, 
the single QD lasing doesn't occur. If the QD position is deviated from the best point, the situation gets worse.

On the other hand, the analyzed whispering gallery modes have $Q_{v}=97,000$ and $V_{\text {mode }}=0.58(\lambda / n)^{3}$. Figure 7 shows $C_{q}$ mapping over the middle slice of PC slabs. It can be noticed that we obtain a more distributed area to put a single QD for this mode. Necessary $Q$-factors, in this case, are $40,000 \times C_{q}$ and $4,200 \times C_{q}(<97,000)$ for homogeneous broadening of $7 \mathrm{meV}$ and $0.7 \mathrm{meV}$, respectively. If we succeed to manipulate single QD position, the single QD lasing is likely to occur. Even if the QD position is deviated slightly from the best point, single QD lasing still may occur due to more distributed $E$ profile in the confined modes. Therefore, single QD lasing at room temperature is possible to occur in $\mathrm{PC}$ nanocavities.

\section{ACKNOWLEDGMENTS}

We appreciate generous supports from the NSF, the AFOSR, and the DARPA.

\section{REFERENCES}

1. E. M. Purcell, "Spontaneous emission probabilities at radio frequencies," Phys. Rev. 69, p. 681, 1946.

2. E. Yablonovitch, "Inhibited spontaneous emission in solid-state physics and electronics," Phys. Rev. Lett. 58, pp. 2059-2062, 1988.

3. S. John, "Strong localization of photons in certain disordered dielectric superlattices," Phys. Rev. Lett. 58, pp. 2486-2489, 1988.

4. O. J. Painter, R. K. Lee, A. Scherer, A. Yariv, J. D. O'Brien, P. D. Dapkus, and I. Kim, "Two-dimensional photonic band-gap defect mode laser," Science 284, pp. 1819-1821, 1999.

5. M. Lončar, T. Yoshie, A. Scherer, Y. Qiu, and P. Gogna, "Low-threshold photonic crystal laser," Appl. Phys. Lett. 81, pp. 2680-2682, 2002.

6. K. Okamoto, M. Lončar, T. Yoshie, A. Scherer, Y. Qiu, and P. Gogna, "Near-field scanning optical microscopy of photonic crystal nanocavities," Appl. Phys. Lett. 82, pp. 1676-1678, 2003.

7. T. Yoshie, J. Vučković, A. Scherer, H. Chen, and D. Deppe, "High quality two-dimensional photonic crystal slab cavities," Appl. Phys. Lett. 79, pp. 4289-4291, 2001.

8. T. Yoshie, O. B. Shchekin, H. Chen, D. G. Deppe, and A. Scherer, "Quantum dot photonic crystal lasers," Electron. Lett. 38, pp. 967-968, 2002.

9. A. Yariv, Quantum Electronics, 3rd Edition, Wiley, New York, 1988.

10. P. G. Eliseev, H. Li, A. Stintz, G. T. Liu, T. C. Newell, K. J. Malloy, and L. F. Lester, "Transition dipole moment of inas/ingaas quantum dots from experiments on ultralow-threshold laser diodes," Appl. Phys. Lett. 77, pp. 262-264, 2000.

11. D. Meschede, H. Walther, and G. Muller, "One-atom maser," Phys. Rev. Lett. 54, pp. 551-554, 1985. 